\title{
Path Loss Correction for Signal Propagation amongst Low Roof Top Buildings using Fuzzy Logic
}

\author{
Sumit Joshi \\ Assistant Professor \\ GRD-IMT, Dehradun \\ M-IAENG
}

\author{
Govind Sati \\ Post Grad. Scholar \\ DIT-U, Dehradun \\ M-IAENG
}

\author{
Mukesh Chandra Kestwal \\ Post Grad. Scholar \\ BTKIT \\ Dwarahat
}

\begin{abstract}
Performance of current path attenuation prediction models encounters huge deviation from their true behavior when deployed for the locality apart from the one for which it had been proven for. This work deals with introducing the path loss on the basis of measured data and representation of the same in a different approach for the mentioned Fuzzy Inference system based analysis. The empirical data collection followed by curve-fitting for path loss evaluation on decibel scale with Normal random variable distribution for representing the shadow fading. Our paper introduces a new methodology for prediction of path loss for betterment in QoS via. Network planning specifically for mobility prone communication systems deploying fuzzy approach. The Transmission discontinuities encountered during propagation has been differentiated in to a variety of factors defined as fuzzy sets such as free space, flat terrain, low foliage terrain, high foliage terrain, and country side terrain. path loss exponent (n) has been applied for varied propagation profiles, Mamdani Fuzzy Inference has been deployed for prediction of " $n$ " path loss exponent for any kind of scenario, which was obtained on the basis of set of symbolic rules that avails an approximation to the known propagation scenarios. Bertoni's model proposed by H.L. Bertoni's has been used for the present analysis.
\end{abstract}

\section{Keywords}

Path loss measurement, Path loss predication, Fuzzy Inference, Fuzzy Modeling.

\section{INTRODUCTION}

Fundamental phenomenon justifying radio propagation are different and hence they are specified by the popular physical properties like: reflection, refraction, path loss, fading, scattering and shadowing for radio signals. Path loss is defined as attenuation in the information signal's strength during propagation from source to the destination. Generally, the path loss varies proportionally with frequency and distance [5].

$$
P L \propto D^{-n}
$$

Gradual and slow variation of signal strength around its average value is called as shadowing where as the rapid variation in the received message signal strength due to multipath propagation is referred to as fading. Accuracy in path loss prediction becomes prominently important better network planning and thus imparting better QoS. Performance and accuracy of any path loss prediction scheme is justified upon data sets such as: Foliage Cover, Residential Cover, and Atmospheric viabilities along with other factors like height of base station, height of mobile and orientation of street angle and many more factors of concern preferably important for network planning now a days.
Different types of outdoor propagation models are used and deployed for determining path loss over variable profile features. Generally, all these different models are used with specific idea of predicting signal strength at some definite receiving point or in a desired location of interest (called zone/sector), the methods may vary in their approach of utility in terms of factors like compatibility and reliability. Most of these models are fundamentally laid down on a well defined algorithmic perception of measured data obtained from empirical test drives [5]. However reliability of these models remains a concern specific to the environment other than for which they have been designed. Measurement drives considering path loss in the practical scenarios and thus their result may be applied to existing models for betterment in reliability of the same [1]. Both theoretical measurement based propagation models indicate that average received signal power decreases logarithmically with distance, whether in outdoor or indoor radio propagations. The average largescale path loss for an arbitrary Tx-Rx distance separation is made directly proportional to the distance by using a path loss exponent " $n$ " as mentioned below:

$$
P L(d) \alpha\left(\frac{d}{d 0}\right)^{n}
$$

$P L(d B)=P L(d 0)+10 n \log (d / d 0)$

Where ' $n$ ' denotes the path loss exponent, depicting the variability in the link loss with respect to the distance, $\mathrm{d} 0$ termed as reference distance and $\mathrm{d}$ referred as $\mathrm{Tx}-\mathrm{Rx}$ separation [5]. Value of this exponent ' $n$ ' depends on fixed propagation scenario, as for free space $n=2$ and for discontinuities in the propagation path ' $n$ ' attains a larger value. The reference distance must always lie in the far field of the radiation pattern radiating from the element such that that near field may not impact the reference path loss. A fundamentally accepted value of $1 \mathrm{Km}$ for macro cell system is used, $100 \mathrm{~m}$ in micro cell systems and $1 \mathrm{~m}$ in Pico cell systems.

The Bertoni's model [18] mentioned in the literature presented an empirical formula for prediction of path loss [9].

In this paper an empirical path loss model for a typical suburban city of India has been proposed. (Patel Nagar, Dehradun). 


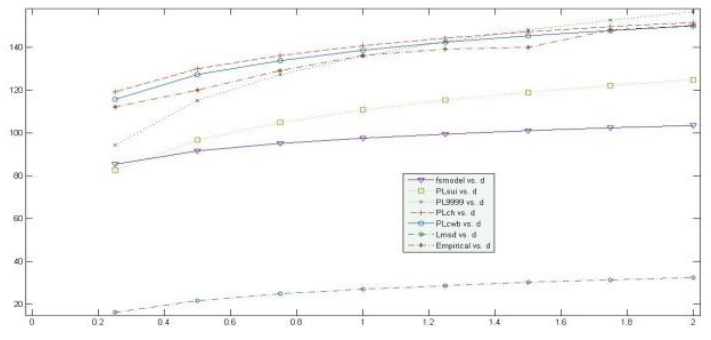

Fig.1.Cumulative comparison of path loss attributed to different models with respect to measured data path loss.

Empirical drive test were performed out in the suburban region of Patel Nagar, Dehradun of its GSM based System. The developed model is also compared with Bertoni's model, which is widely used for path loss prediction in scenarios with low roof top residential outlook.

\section{BERTONI MODEL}

This model is most suitable for flat sub-urban \& urban areas with uniform building height. Among other models this model gives a more precise path loss. This is a result of additional parameters introduced which characterize different environments. It also covers distinguishing on basis of terrain features. The path loss equation for the same is given as:

For LOS condition

$P L(L O S)=42.6+26 \log (d)+20 \log (f)$

For N-LOS condition, it follows as:

$\mathrm{PL}_{\mathrm{LOS}}=\left\{\mathrm{L}_{\mathrm{FSL}}+\mathrm{L}_{\mathrm{rts}}+\mathrm{L}_{\mathrm{msd}} \quad\right.$,for urban \& sub-urban

$$
\left.\mathrm{L}_{\mathrm{FS}} \quad \text {,if } \mathrm{L}_{\mathrm{rts}}+\mathrm{L}_{\mathrm{msd}}>0 \quad\right\}
$$

Where,

$\mathrm{L}_{\mathrm{FSL}}=$ Free space loss

$\mathrm{L}_{\mathrm{rts}}=$ Roof top to street diffraction

$\mathrm{L}_{\mathrm{msd}}=$ Multi screen diffraction loss

11 material on each page should fit within a rectangle of $18 \mathrm{x}$ $23.5 \mathrm{~cm}(7 " \mathrm{x} 9.25 ")$, centered on the page, beginning $2.54 \mathrm{~cm}$ (1") from the top of the page and ending with $2.54 \mathrm{~cm}(1 ")$ from the bottom. The right and left margins should be $1.9 \mathrm{~cm}$ (.75"). The text should be in two $8.45 \mathrm{~cm}(3.33 ")$ columns with a $.83 \mathrm{~cm} \mathrm{(.33")} \mathrm{gutter.}$

This model relies on ray tracing technique considering each building block as a diffraction screen.

The major feature of Bertoni's model is that it deals with path gain instead of path loss parameter which is a common trait in other models, although related to the later as:

PG $\equiv$ Path Gain $=\frac{\text { recieved } \text { power }}{\text { transmitted } \text { power }}$

(PG is always less than 1)
$\mathrm{PL} \equiv$ Path Loss $=\frac{\text { transmitted } \text { power }}{\text { received } \text { power }}$

(PL is always greater than 1)

When expressed in $\mathrm{dB}, P G d B=10 \log P G=-L$

Where $L \equiv 10 \log P L$

If $P=P T A / R^{n}$, then $P G d B=10 \log A-10 \mathrm{nlog}$

$\mathrm{R}$ and $L=-10 \log A+10 n \log R$.

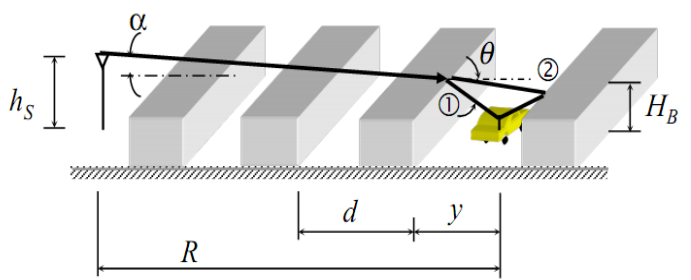

Fig.2.Bertoni model implementation layout for propagation path gain over buildings

Major factors facilitating Bertoni model to be considered are:

1. Low building environment facilitation by deploying uniform radio absorbers array in place of rows of buildings.

2. Street grid organization made aptly suitable by accounting for intra-building spacing and back-toback spacing.

3. Use of simple geometric techniques for lower building scenario and ray-tracing techniques for high-rise building scenario both.

4. Considering propagation as a roof-top phenomenon.

5. Classification of path loss i.e. path gain in to three subtle factors each accounting for free-space loss, diffraction loss due to edges etc. is depicted as physical approximation asFig.3 [2],

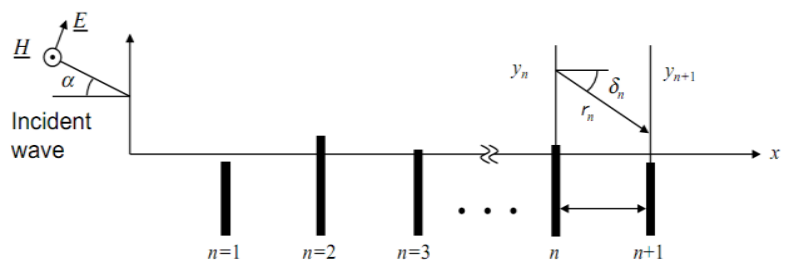

Fig.3. Physical optics approximation for roof-top field reduction

Path Gain

$$
P G=(P G 0)(P G 1)(P G 2)
$$

Where,

$P G 0=\frac{\lambda}{4 \pi r^{2}}[$ free-space path gain $]$

$P G 1=Q 2$ [Reduction in the field at the roof top just before the mobile due to propagation past previous rows of buildings given by a factor Q]. 
$\mathrm{PG}_{2}=\frac{1}{2 \pi k \rho_{1}}\left(\frac{1}{\left|\theta_{1}\right|}-\frac{1}{2 \pi-\left|\theta_{1}\right|}\right)^{2}+\frac{|\Gamma|^{2}}{2 \pi k \rho_{2}}\left(\frac{1}{\left|\theta_{2}\right|}-\right.$ $\left.\frac{1}{2 \pi-\left|\theta_{2}\right|}\right)^{2}$ which accounts for the roof top field down to mobile i.e. summing the ray powers to get the small average.

Where, $\rho_{1}$ and $\rho_{2}$ have their usual meanings as in electromagnetic studies.

Reduction of rooftop fields for a spherical wave incident on the rows of buildings is the same as the reduction for an incident plane wave after many rows. Reduction in the field strength occurs due to multiple forward diffractions past an array of absorbing screens for a plane wave with unit amplitude that is incident at glancing angle $\alpha$ as depicted in Fig.3.earlier.

The physical analogy deployed in Bertoni's model is based on ray tracing techniques and involves replacing buildings by parallel absorbing screens. For parallel screens, the reduction factor is found by repeated application of the Kirchhoff integral. Going from screen $n$ to screen $n+1$, the integration is as:

$\mathrm{H} \quad\left(x_{n+1}, y_{n+1}\right)=\int_{-\infty}^{\infty} \int_{h_{n}}^{\infty}\left(\cos \alpha_{n}+\cos \delta_{n}\right) H\left(x_{n}, y_{n}\right)$

$\frac{j k e^{-j k r}}{4 \pi r} \mathrm{~d} y_{n} \mathrm{~d} z_{n}$

\section{PATH LOSS MODEL BASED ON THE FIELD MEASUREMENTS}

Field measurements in the concerned scenario was performed for which a path loss model is to be developed, this encompasses the merit of deployment of different environmental entities regardless of the fact whether they can be separately resolute. From the measured data, a path loss model is developed by statistical analysis of the data. After performing the field measurement task efficiency of such models becomes highly validated. Field measurements were performed in the suburban region of Patel Nagra; Dehradun for its GSM based system. All the measurements were made for mobile terminal using TEMS-10.1 ASCOM utility rifs.

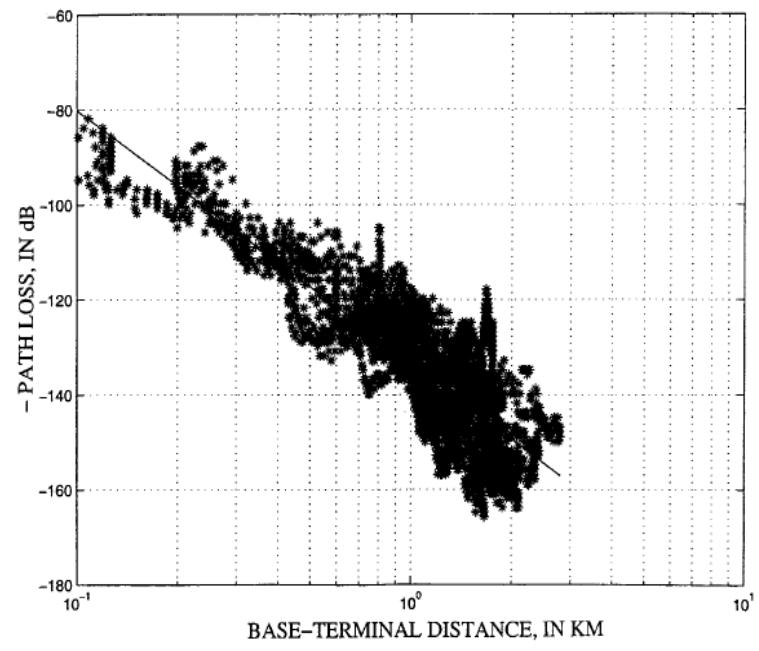

Fig.4. Scatter plot of path loss and distance with respect to base terminal (base antenna height was above $30 \mathrm{~m}$ )
Measurements were taken in only two zones/sectors due to terrain limitations and permissions as further cover comes under military area. For macro cellular system, the reference distance is taken as $\mathrm{d} 0=0.5 \mathrm{~km}$. Starting from $0.5 \mathrm{~km}$, measurements were taken in intervals of $0.5 \mathrm{~km}$ in two of the concerned zones. In all, 3 cellular base stations were involved in the field measurements. For each of these, the test signal was transmitted close to $1800 \mathrm{MHz}$, and the mobile drive test car drove around the cellular coverage area at an angular span of 180 degrees measuring and recording local mean power in two sectors. In The global positioning system (GPS) data were also collected, which made it possible to determine the radial distance from the base station related with each power measurement. The experimental data were taken at distances ranging from 500 meters to $5 \mathrm{~km}$ as the range of clear signal is $2 \mathrm{~km}$ to $5 \mathrm{~km}$ power measured by Spectrum Analyzer at different locations in intervals of $0.5 \mathrm{~km}$ as summarized in Table.1.

The Table. 2 above depicts the measured valued obtained for power received levels obtained during the drive test. The measured values for received power varies with the distance in different sectors, median values for ungrouped frequency distribution of the received power for model formulation can be deployed. The reference path loss Lp (d0) is $112.6 \mathrm{~dB}$. The path loss exponent $\mathrm{n}$ is obtained from the measured data, by linear regression such that the difference between the measured and estimated path loss is minimized in a mean square sense. The sum of squared error is given by:

$\mathrm{E}(\mathrm{n})=\sum_{i=1}^{k}\{L p(d i)-\mathbf{L} p(d i)\} 2$

Where $\mathrm{Lp}$ (di) is the measured path loss at distance di and Ĺp (di) is its estimate using equation (1). The value of $n$, which minimizes the mean square error, is obtained by equating the derivative of equation (12) to zero, and when solving for $\mathrm{n}$.

$d E(n) / d n=0$

The obtained value for $n=4.34$.

Table.1. Empirical Measurements Based Results for 1800 MHz

\begin{tabular}{|c|l|}
\hline $\begin{array}{c}\text { Distance from the } \\
\text { transmitter(in Km) }\end{array}$ & Path Loss (in dB) \\
\hline 0.5 & 112.6 \\
\hline 1 & 118.7 \\
\hline 1.5 & 130.7 \\
\hline 2 & 139.1 \\
\hline 2.5 & 142.2 \\
\hline
\end{tabular}


Table.2. Received Power values using Tems10.1

\begin{tabular}{|c|c|c|c|}
\hline $\begin{array}{c}\text { Distance } \\
\text { from } \\
\text { transmitter d } \\
(\mathbf{~ k m})\end{array}$ & \multicolumn{2}{|c|}{$\begin{array}{c}\text { Received power in } \\
\text { different } \\
\text { zones, (dBm) }\end{array}$} & $\begin{array}{c}\text { Median value, } \\
(\mathbf{d B m})\end{array}$ \\
\hline & $\alpha$ & $\beta$ & Mean Value \\
\hline 1.0 & -74 & -72 & -73 \\
\hline 1.5 & -77 & -75 & -76 \\
\hline 2.0 & -79 & -77 & -78 \\
\hline 2.5 & -81 & -79 & -80 \\
\hline
\end{tabular}

\section{MAMDANI FUZZY INFERENCE PATH LOSS MODELS}

Mamdani Fuzzy inference system is based on fuzzy logic science, which rationalizes uncertainty in events to make it predictable. It considers vague concepts and provides a logical output for same. Fuzzy Logic has been extensively used in many applications where precise mathematical modeling is not feasible [7,8]. It enables us to utilize the concept of Fuzzy logic to characterize an undefined propagation scenario from a set of available scenario sets. This concept has been illustrated as Fig.5 where the propagation has been featured into several propagation scenarios defined as an input fuzzy set such as X1 $=$ Residential cover and X2 = Foliage cover. These crisp input sets are firstly by fuzzified using some fuzzifier entity to fuzzy sets and then developed to be used along with fuzzy rule base leading to a fuzzy output. To obtain crisp output sets, a method call "de-fuzzification" is used to extract a crisp value that best represents the link loss values.

Fuzzy logic reasoning is then put to action for determining the link slope for a propagation scenario which is unknown by its nature but do closely approximate one of the available environments scenarios.
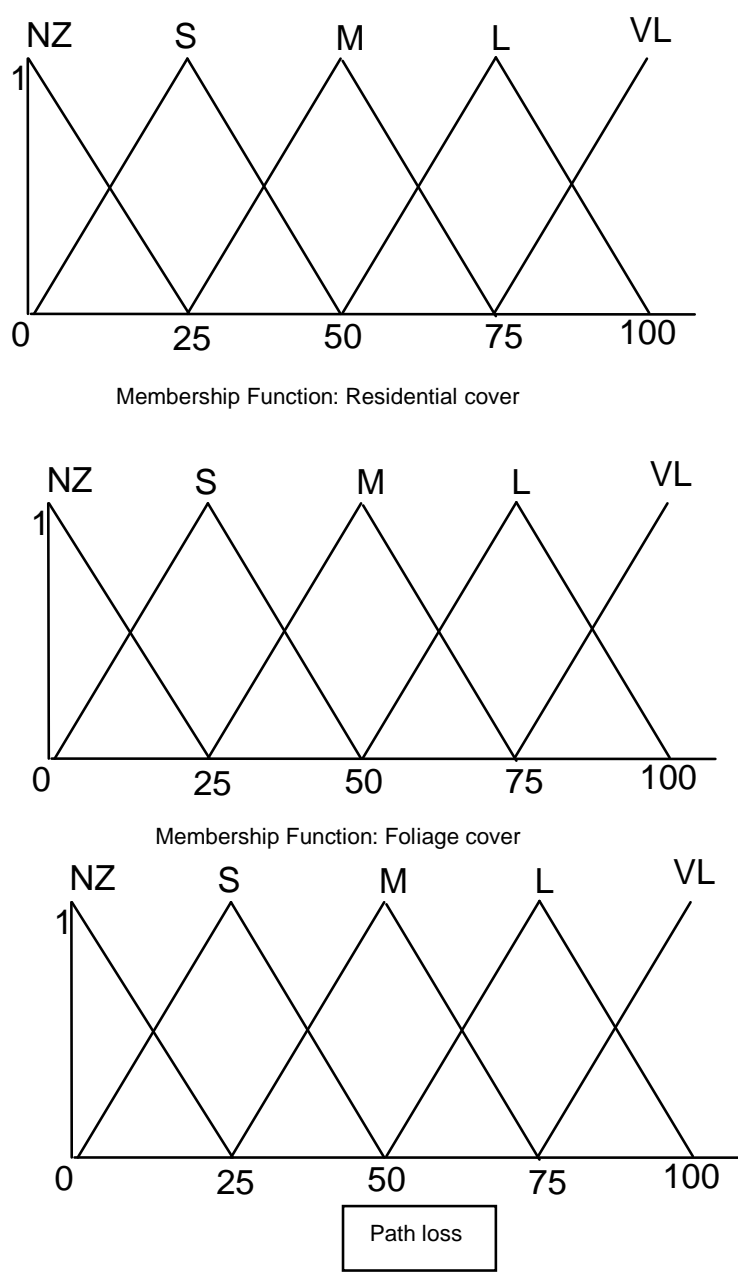

Fig.5. Fuzzy Linguistic Membership Functions

We have used triangular membership functions and classified the fuzzy variable into 5 levels as NZ: Nearly zero, S: Small, M: Medium, L: Large, and VL: Very large.

The different input scenario based variables may be classified as:

For input X1, Residential cover,

Very large $=$ Dense Urban: Down-town having high rise buildings and very high mass.

Large $=$ Urban: Down-town having high rise buildings on both sides of the vegetations.

Medium = Suburban: Just outside of down-town area, residential areas.

Small = Rural: Open area, roads and highways, having no residential areas.

Nearly zero $=$ Free Space: A propagation environment having no obstructions.

For input X2, Foliage covers,

Very large vegetation mass: Virgin forest

Large vegetation mass: Thick forest, timber forest. 
Medium vegetation mass: Public Park, zoo garden.

Small vegetation mass: Herb-garden, flower garden, dwarf canopy.

Nearly zero $=$ Free Space: A propagation environment having no vegetation.

The scenarios to be predicted may be obtained by means of the following fuzzy Rule sets:

$$
\begin{aligned}
& \text { Rule1: if } \mathrm{X} 1=\mathrm{VL} \text {, then } \mathrm{Y} \rightarrow 3 \\
& \text { Rule2: if } \mathrm{X} 1=\mathrm{L}, \text { then } \mathrm{Y}=\mathrm{L} \\
& \text { Rule3: if } \mathrm{X} 1=\mathrm{M}, \text { then } \mathrm{Y}=\mathrm{M} \\
& \text { Rule4: if } \mathrm{X} 1=\mathrm{S}, \text { then } \mathrm{Y}=\mathrm{S} \\
& \text { Rule5: if } \mathrm{X} 1=\mathrm{NZ} \text {, then } \mathrm{Y} \rightarrow 2 \\
& \text { Rule6: if } \mathrm{X} 2=\mathrm{VL} \text {, then } \mathrm{Y} \rightarrow 4 \\
& \text { Rule7: if } \mathrm{X} 2=\mathrm{L}, \quad \text { then } \mathrm{Y}=\mathrm{L} \\
& \text { Rule8: if } \mathrm{X} 2=\mathrm{M}, \text { then } \mathrm{Y}=\mathrm{M} \\
& \text { Rule9: if } \mathrm{X} 2=\mathrm{S}, \quad \text { then } \mathrm{Y}=\mathrm{S} \\
& \text { Rule10: if } \mathrm{X} 2=\mathrm{NZ} \text {, then } \mathrm{Y} \rightarrow 1 \\
& \text { Rule11: if } \mathrm{X} 1=\mathrm{M} \text {, and } \mathrm{X} 2=\mathrm{L} \text {, then } \mathrm{Y}=\mathrm{L} \\
& \text { Rule12: if } \mathrm{X} 1=\mathrm{M} \text {, and } \mathrm{X} 2=\mathrm{M} \text {, then } \mathrm{Y}=\mathrm{M} \\
& \text { Rule13: if } \mathrm{X} 1=\mathrm{M} \text {, and } \mathrm{X} 2=\mathrm{S} \text {, then } \mathrm{Y}=\mathrm{M}
\end{aligned}
$$

Apparently, the above linguistic rule provides a fine tuning of propagation environments which have already been established experimentally. Now, we implement the above rules to find the output results. Fuzzy output value has very little practical use as most application requires non fuzzy (crisp) control actions therefore it is necessary to produce a crisp value to represent the possibility distribution of the output using defuzzification. Sum of means method for defuzzification is used for the present analysis can be expressed as:

$$
\begin{gathered}
f(y)=\sum \mu(y) \cdot y \\
\sum \mu(y)
\end{gathered}
$$

and applying Sum of means method we obtain:

$$
f(y)=\sum_{m=1}^{n} E^{m} D^{m}
$$

Where:

$\mathrm{f}(\mathrm{y})$ is the crisp output value

$\mathrm{E}^{\mathrm{m}}$. is the crisp weighting for the linguistic value $\mathrm{LV}^{\mathrm{m}}$

$\mathrm{D}^{\mathrm{m}}$ is the membership value of $\mathrm{y}$ with relation to the linguistic value $\mathrm{LV}^{\mathrm{m}}$

\section{RESULTS}

The experimental data were analyzed to find path loss slope for each terrain by linear regression. The results obtained by using fuzzy logic approximation are shown in Table-3.
Table.3. Values for Link Loss Slope using Fuzzy inference

\begin{tabular}{|l|c|}
\hline Terrain Type & $\begin{array}{l}\text { Fuzzy } \\
\text { (n) }\end{array}$ \\
\hline Clear Area & 2.2 \\
Light Vegetation & 3.3 \\
Small Town & 4.1 \\
Heavy Vegetation & 4.7 \\
\hline
\end{tabular}

\section{CONCLUSION}

Path loss models based on measured data have been presented using linear regression and Fuzzy logic. The models are based on a simple dn exponential path loss Vs. distance relationship.

It has been shown from the above considerations and relations; the fuzzy logic set approach to path loss prediction puts a new method for communication analysis in complex system, which RF propagation is chaotic in multipath environment owing to numerous RF barriers and scattering phenomena from several objects in the environment.

\section{REFERENCES}

[1] A.A.Moinuddin and S Singh, "Accurate Path Loss Prediction in Wireless Environment", The Institution of Engineers (India) Volume 88, July 2007, Pp: 0913Bowman, M., Debray, S. K., and Peterson, L. L. 1993. Reasoning about naming systems.

[2] S. Joshi, V. Gupta, "A Review on Empirical Data Collection and Analysis of Bertoni's Model at 1.8 GHz,IJCA, Vol.56 No.6, October, 2012, Pp: 17-23.

[3] C. Tornevik, J.E. Berg, F. Lotse and M. Madfors, "Propagation Cell Planning and Channel Allocation for Indoor Application of Cellular System", IEEE Veh. Techno., Vol.48, May 1993, Pp. 867-870.

[4] Dharm Prakash Agarwal and Qing-An Zeng, "Introduction to Wireless and Mobile Systems", Thomson and Brooks/Cole, 2003, Pp: 63-87.

[5] Faruque, Saleh, "Propagation Prediction Based on Environmental Classification and Fuzzy Logic Approximation”, Proc. IEEE ICC'96, 1996, Pp 272-276.

[6] G L Stuber., "Principles of Mobile Communications", Kluwer Academic Publishers, 1996, Pp: 162-198.

[7] Kosko, Bart, and Satoru Isaka, "Fuzzy Logic", Scientific American, July 1993, Pp.76-81.

[8] Kosko,Bart, "Fuzzy Thinking”, New York: Hyperion, 1993.

[9] S. Joshi, V. Gupta and S. Vijay ,"Model Selection for Radio Propagation in Suburban Niche with Low Roof Residential Scenario", .IJCA ,Vol 57,No 4 ,November 2012,Pp 39-42.

[10] M. M. Peritsky, "Statistical estimation of mean signal in a rayleigh-fading environment",IEEE Trans. Veh. Technology., vol. VT-22, pp. 123-129, Nov. 1973

[11] R. A. Valenzuela, O. Landron, and D. L. Jacobs, "Estimating Local Mean Signal Strength of Indoor 
Multipath Propagation", IEEE Transaction on vehicular technology, Vol.46, No.1, February, 1997

[12] Scott Y. Seidel and T. S. Rappaport, “ 914 MHz Path Loss Prediction Models for Indoor Wireless Communications in Multi floored Buildings.", IEEE Trans. Antennas Propagation., vol. 40, pp. 207-217, Feb, 1992.

[13] S. Y. Seidel, T. S. Rappaport, "Site specific propagation prediction for wireless in building communication system design", IEEE Transaction on vehicular technology, Vol.43, Nov. 1994, pp. 879 - 891

[14] T. S. Rappaport, S. Y. Seidel, and K. R. Schaubach, “ Site-specific propagation prediction for PCS system design", in Virginia Tech's Second Symp Wireless Personal Comm., Blacksburg, VA, June 17 - 19, 1992, pp. $16.1-16.27$

[15] T. S. Rappaport., "Characterization of UHF multipath radio channels in factory buildings", IEEE Trans. Antennas Propagation., vol. 37, pp. 1058-1069, Aug, 1989

[16] T S Rappaport "Wireless Communication Principles and Practice", Pearson Education Pvt. Ltd 2002.

[17] Vishal Gupta, Sandip Vijay and S.C.Sharma, "Efficient Path Loss Prediction in Mobile Wireless Communication Network", ISN2008, Feb. 22-24, 2008, Pg-271.

[18] J.S Seybold, "Introduction to RF Propagation", John Wiley, 2005.

[19] W.C.Y.Lee, "Mobile Communication Design Fundamentals", ( $2^{\text {nd }}$ Ed), John Wiely\&Sons, New York, 1993.

\section{MAIN AUTHOR'S PROFILE}

Mr. Sumit Joshi became a Member (M) of IAENG in 2011. He presently lives in Dehradun and completed his Graduation as a B.Tech graduate in the field of Electronics \& Communications from Dehradun Institute of Technology, Dehradun, Uttarakhand, India. He did his post-graduation as an M.tech scholar in the field of Digital Communications from the same in 2013. Presently he is working on 'radio propagations at $1.8 \mathrm{GHz}$.'. $\mathrm{He}$ is working at GRD-IMT, Dehradun at present as an assistant professor for sharing his knowledge with undergraduate students, where he instructs students regarding digital signal processing techniques, telecommunication switching, matlab tools \& techniques, qualnet basics, HFSS, Radio plan \& Neural networks, electromagnetic fields \& radar and navigation basics. He is also a member of some of the prestigious associations like ISOC \& IJOE. He has published many papers in refereed international and national journals and has supervised many projects.

Mr. Govind Sati became a Member (M) of IAENG in 2013. He presently lives in Dehradun and completed his Diploma in the field of Electronics Engineering from Govt. Poly. Gauchar, Chamoli, Uttarakhand, India in 2009. Then he completed his Graduation as a B.Tech graduate in the field of Electronics \& Communications from Ghaziabad Institute of Management \& Technology, Ghaziabad, UP, India in 2012. $\mathrm{He}$ is a post-graduate scholar in the field of Wireless \& Mobile Communications from Dehradun Institute of Technology, Dehradun, Uttarakhand, India in 2013. Presently he is working on 'radio propagations at $2.1 \mathrm{GHz}$." 